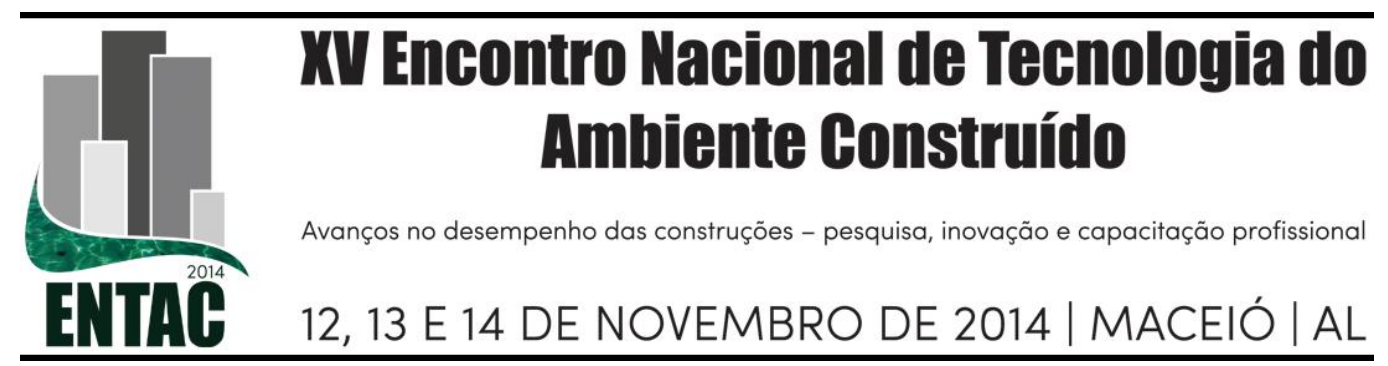

\title{
COMPORTAMENTO DAS MISTURAS DE AGREGADO RECICLADO-SOLO PARA FINS GEOTÉCNICOS
}

MACEDO, Thaísa (1); LAFAYETTE, Kalinny (2); BEZERRA, Jonas (3)

(1) ESUDA, e-mail: thaisa_197@hotmail.com; (2)UPE, e-mail: klafayette@gmail.com; (3) UPE, jonas.silva.eng@gmail.com

\begin{abstract}
RESUMO
O Brasil, nos últimos anos, tem crescido bastante no setor imobiliário, e este tem uma parcela significativa para a economia do país. Isto é muito importante, entretanto a construção civil é um setor que afeta o meio ambiente, devido à "exploração" de recursos naturais, desperdício de materiais nas obras, entre outros. A perda de materiais junto ao descarte irregular dos resíduos da construção civil (RCC), torna-se um desafio para a indústria da construção criar novos mecanismos para reaproveitar esses materiais e dar soluções de aplicações. Com este intuito, foi realizada uma pesquisa experimental, com o objetivo de avaliar o comportamento dos diferentes percentuais de agregado reciclado no compósito com solo através de ensaios compactação, de Índice de Suporte Califórnia (ISC) e da avaliação da quebra dos grãos por meio dos índices de degradação. Os materiais utilizados foram o agregado reciclado e o solo. Com estes materiais, foi possível realizar os ensaios de caracterização física (granulometria, densidade real, limites de Atterberg), ensaios de forma dos grãos com o agregado reciclado e mecânicos (compactação e índice de suporte Califórnia) com as misturas, verificando também a quebra dos grãos pós-ISC com e sem imersão. Os resultados de granulometria mostraram que à medida que o percentual de agregado reciclado na mistura aumentava, o material se tornava mais granular, com maiores percentuais de areia. Os valores de ISC dos materiais, com e sem imersão, mostraram que a presença do agregado reciclado na mistura fez aumentar o ISC e esse acréscimo resultou em um incremento de ISC superior a cinco vezes (com imersão) e seis vezes (sem imersão) em relação ao valor de ISC do solo. Conclui-se que as amostras utilizadas apresentam características físicas e mecânicas que permitem seu uso em substituição aos materiais convencionais para fins geotécnicos, como em camadas de pavimentos.
\end{abstract}

Palavras-chave: Agregado reciclado, Índice de Suporte Califórnia, camadas de pavimentos.

\begin{abstract}
Brazil in recent years has grown considerably in real estate, and this has a significant part to the country's economy. This is very important, however the civil construction is an industry that affects the environment, due to the "exploitation" of natural resources, waste of materials in the works, among others. The loss of material along the irregular disposal of civil construction waste (CCW) becomes a challenge for the construction industry to create new mechanisms to recycle these materials and provide application solutions. For this purpose, an experimental study was performed to evaluate the behavior of different percentages of recycled aggregate in the composite with soil through compaction tests, California Bearing Ratio (CBR) and the evaluation of kernel cracking through degradation rates. The used materials were recycled aggregate and soil. With these materials, it was possible to perform physical characterization tests (particle size, true density, Atterberg limits), testing of grain shape with recycled aggregate and mechanical tests (compaction and California Bearing Ratio) with the composites, also checking the grains breakdown post- CBR, with and without immersion. The particle size results showed that as the percentage of recycled aggregate in the composite increased, the material became more granular, with higher percentages of sand. The CBR values of materials, with and without immersion showed that the presence of recycled aggregate in the composite increased the CBR and this increase has resulted in an increasement of more than five times (with immersion) and six times (without immersion) compared to the soil CBR value. It was concluded that the samples used have physical and
\end{abstract}


mechanical characteristics that allow its use in place of conventional materials for geotechnical purposes as in pavement layers.

Keywords: recycled aggregate, California bearing ratio, pavement layers.

\section{INTRODUÇÃO}

A indústria da construção civil tem um papel muito importante na economia de um país, pois através das construções de escolas, hospitais, residências, centros comerciais, entre outras, é que as cidades vão crescendo e ganhando "novos" espaços.

Tal desenvolvimento também é advindo do crescimento da população e do surgimento de novos empreendimentos atendendo, desta forma, as diferentes classes e contribuindo para o desenvolvimento das cidades.

Ao mesmo tempo em que as cidades "crescem”, os resíduos também são produzidos, devendo ser dada uma atenção maior, pois os resíduos da construção civil (RCC) ocupam bastante espaço no meio ambiente.

A geração de RCC no âmbito nacional está associada à falta de planejamento entre as fases de projeto e execução das obras, trazendo com isso, uma maior probabilidade de mudanças durante a fase de execução (CORNELI, 2009). Além disso, a aplicação dos materiais é realizada de forma tradicional, ou seja, rudimentar com técnicas simples que contribuem para o desperdício dos materiais e a geração de resíduos (GUSMÃO, 2008).

Muitas vezes esses RCC são descartados em locais inapropriados como em córregos de rios, terrenos baldios, canais, encostas, beira de estradas, podendo surgir vetores de doenças quando da presença de resíduo doméstico.

Essa é uma situação muito preocupante, com isto, é tão importante a realização de estudos que possa reaproveitar estes resíduos, evitando a poluição do meio ambiente e o esgotamento dos aterros sanitários, ou até mesmo a construção de novos aterros.

A utilização do agregado reciclado na própria construção civil, através de várias pesquisas, mostra que é um material com um bom potencial de uso em diversas situações, como: base e sub-base para pavimentação; agregados para concreto; matériaprima para cerâmica de revestimento, etc.

Com base nisso, a presente pesquisa visa o reaproveitamento do RCC em forma de agregado reciclado com a introdução de solo, com diferentes percentuais, formando uma mistura ou compósito para aplicações com fins geotécnicos, como por exemplo, em camadas de pavimentos.

\section{MATERIAIS}

As amostras de resíduos da construção civil (RCC), já beneficiada, pela Usina de Beneficiamento - Ciclo Ambiental. Esta Usina está localizada no município de Camaragibe-PE, Região Metropolitana do Recife-PE.

$\mathrm{O}$ agregado reciclado (AR) coletado foi, aproximadamente, de $600 \mathrm{~kg}$ e transportado para o laboratório da Escola Politécnica de Pernambuco, onde foi peneirado para garantir dimensões menores a $4,8 \mathrm{~mm}$ e, posteriormente, serem submetidos aos ensaios de caracterização física e mecânica.

Foram coletados aproximadamente $450 \mathrm{~kg}$ de amostras de solo no Parque Armando de Holanda Cavalcanti, situado no município do Cabo de Santo Agostinho-PE. Após a 
coleta, o solo foi levado ao laboratório da Escola Politécnica de Pernambuco e armazenado para posterior caracterização e realização dos ensaios.

O solo Residual de Granito foi escolhido com base em outros estudos de Silva et al. (2013) e Lafayette (2006), por exemplo, pela facilidade de obtenção do material in loco. Durante a coleta descartou-se o solo com a presença de materiais orgânicos, para não comprometer a qualidade da amostra e dos resultados dos ensaios.

As misturas são formadas pelo solo e pelo agregado reciclado com diferentes percentuais. Logo, o Quadro 1 apresenta a relação de como foi identificada cada amostra pura e as misturas. Além disso, a quantidade de solo e agregado reciclado foi determinada em relação à massa seca da mistura de agregado reciclado-solo.

Quadro 1 - Identificação das amostras.

\begin{tabular}{|c|}
\hline Amostra \\
\hline *AR $(100 \%)$ \\
\hline Solo $(100 \%)$ \\
\hline AR $(25 \%)+$ Solo \\
\hline AR $(50 \%)+$ Solo \\
\hline AR $(75 \%)+$ Solo \\
\hline
\end{tabular}

$* \mathrm{AR}=$ agregado reciclado

Fonte: Autor

\section{MÉTODOS}

Para ter uma organização na elaboração dos ensaios, foram ordenados os ensaios, seguindo estas etapas:

- $1^{\text {a }}$ etapa: Ensaios de caracterização física (granulometria, densidade real, limites de atterberg e forma dos grãos).

- $2^{\mathrm{a}}$ etapa: Ensaios mecânicos (compactação e Índice de Suporte Califórnia).

\subsection{Caracterização física}

Os ensaios de granulometria tiveram como base a norma NBR 7181 (ABNT, 1984), em que foi possível determinar as curvas granulométricas das amostras puras e das misturas.

Quanto aos ensaios de densidade real, utilizou-se a norma NBR 6508 (ABNT, 1984), com o uso das bombas à vácuo e do picnômetro, realizando três vezes para cada amostra, tendo diferenças mínimas.

Também foram realizados os ensaios de limites de Atterberg para todas as amostras, em que determinou-se a plasticidade dos materiais. Para o limite de plasticidade empregouse a norma NBR 7180 (ABNT, 1984), já para a obtenção do limite de liquidez utilizouse a norma NBR 6459 (ABNT, 1984).

O ensaio de forma dos grãos foi realizado conforme a norma NBR 6954 (ABNT, 1989) apenas para o agregado reciclado. Através dessa norma, é possível a determinação da forma para o material lastro padrão utilizada em via férrea. Esse ensaio é feito com auxílio de um paquímetro, ou seja, um instrumento utilizado para medir comprimento (a), largura (b) e altura (c) dos grãos. Por meio dessa medição, o material pode ser classificado em diferentes formas, como mostra o Quadro 2. 
Quadro 2 - Classificação da forma dos grãos.

\begin{tabular}{|c|c|}
\hline Método das relações b/a e c/b & Forma \\
\hline $\mathrm{b} / \mathrm{a}>0,5 \mathrm{e} \mathrm{c} / \mathrm{b}>0,5$ & Cúbico \\
\hline $\mathrm{b} / \mathrm{a}<0,5 \mathrm{e} \mathrm{c} / \mathrm{b}>0,5$ & Alongado \\
\hline $\mathrm{b} / \mathrm{a}>0,5 \mathrm{e} \mathrm{c} / \mathrm{b}<0,5$ & Lamelar \\
\hline $\mathrm{b} / \mathrm{a}<0,5 \mathrm{e} \mathrm{c} / \mathrm{b}<0,5$ & alongado-lamelar \\
\hline
\end{tabular}

Fonte: NBR 6954 (ABNT, 1989)

Para a verificação da forma dos grãos, foram utilizados, aproximadamente, 2,0 kg de material, retidos nas peneiras de $25,4 \mathrm{~mm}, 19,1 \mathrm{~mm}, 9,52 \mathrm{~mm}, 4,76 \mathrm{~mm}$ e 2,0 $\mathrm{mm}$. A Figura 1 apresenta a medição das partículas.

\section{Figura 1 - Obtenção da forma dos grãos do agregado reciclado.}

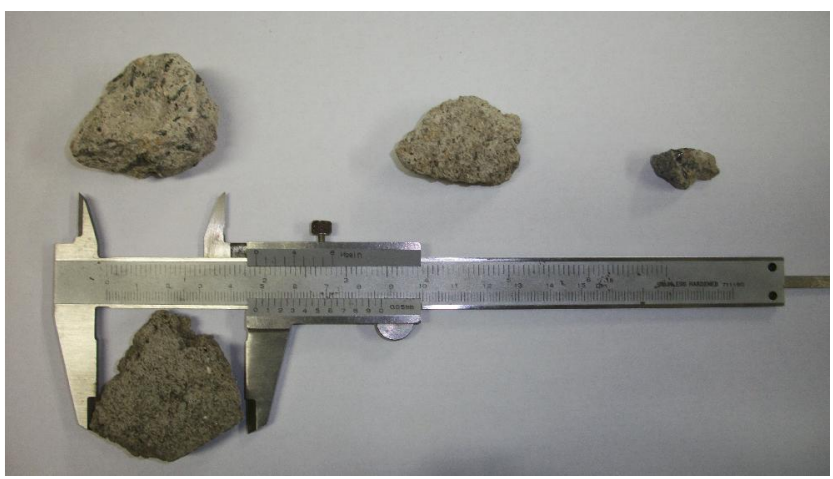

Fonte: Autor

\subsection{Caracterização mecânica}

A umidade ótima e o peso específico máximo foram obtidos a partir do ensaio de compactação com as amostras de solo, agregado reciclado e das misturas, baseando-se na norma brasileira NBR 7182 (ABNT, 1986), onde foi utilizada a energia de Proctor Intermediária.

Os ensaios de compactação foram importantes para a verificação das prováveis mudanças das curvas granulométricas após serem realizados os ensaios de ISC e determinar os índices de degradação das amostras.

Para a realização da análise da quebra dos grãos, utilizou-se os procedimentos na norma DNER-ME 398/99. São determinadas as porcentagens de material que passa em seis peneiras antes e após a compactação, realizando a subtração entre as porcentagens inicial e final (D), como mostra a equação (1):

$$
\mathrm{ID}_{\mathrm{p}}=\frac{\Sigma \mathrm{D}}{6}
$$

O Índice de Degradação (IDp) corresponde à soma das diferenças que passam por cada peneira, dividida pela quantidade de peneiras utilizadas $(12,5 \mathrm{~mm}-1 / 2$ " ; $9,5 \mathrm{~mm}-3 / 8$ "; $4,75 \mathrm{~mm}-\mathrm{n}^{\mathrm{o}} 4 ; 2,0 \mathrm{~mm}-\mathrm{n}^{\mathrm{o}} 10 ; 0,425 \mathrm{~mm}-\mathrm{n}^{\mathrm{o}} 40$ e $\left.0,075 \mathrm{~mm}-\mathrm{n}^{\mathrm{o}} 200\right)$.

Além do índice de degradação IDp existe também outra forma de obter a análise da degradação dos grãos, através do Índice de degradação de Ruiz, que é obtido a partir do somatório dos deslocamentos $\Delta \mathrm{i}$ da curva granulométrica em cada peneira, dividido pelo $\mathrm{n}^{\mathrm{o}}$ de peneiras usadas no ensaio (Buzatti, 1987 apud Valle, 1994). O índice é obtido através da equação (2): 


$$
\mathrm{ID}=\frac{\sum \Delta \mathrm{i}}{\mathrm{N}}
$$

onde, ID = Índice de Degradação de Ruiz

$\mathrm{n}=\mathrm{n}^{\mathrm{o}}$ de peneiras utilizadas no ensaio

$\Delta \mathrm{i}=$ Deslocamento da curva granulométrica

Para o ensaio de Índice de Suporte Califórnia (ISC), utilizou-se a norma NBR 9895 (ABNT, 1987). A prensa manual neste ensaio tem capacidade máxima de 5000kgf para a penetração nos corpos de prova e foi utilizada a velocidade do pistão padrão de $1,27 \mathrm{~mm} / \mathrm{min}$.

\section{RESULTADOS}

\subsection{Caracterização física}

\subsubsection{Granulometria e índices físicos}

A partir das curvas granulométricas (Figura 2) foi possível observar que o solo e a maior parte das misturas não possuem coeficientes de uniformidade. Em contrapartida, a mistura de $\mathrm{AR}(75 \%)+$ Solo apresentou $\mathrm{Cc}=12$ e $\mathrm{Cu}=1,74$. Além disso, todas as amostras possuem dimensões inferiores a $63,5 \mathrm{~mm}$, seguindo o que a norma NBR 15115 (ABNT, 2004).

Figura 2 - Curvas granulométricas das amostras.

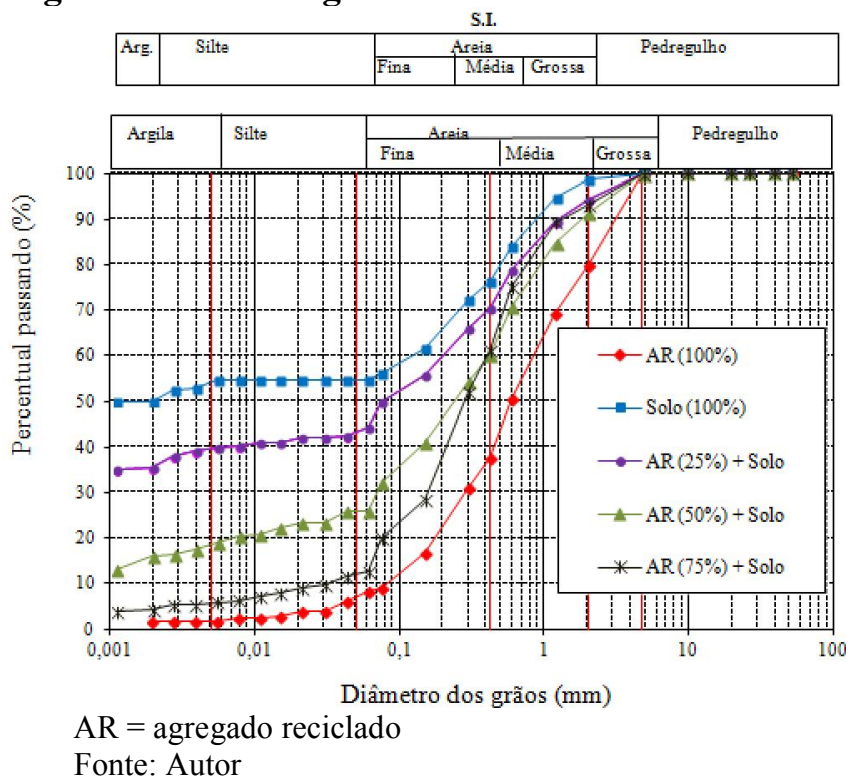

Os percentuais de pedregulho, areia, silte e argila estão dispostos na Tabela 1, onde ficou claro o aumento de material mais granular a medida que o percentual de agregado reciclado também aumentava. 
Tabela 1 - Percentual de argila, silte, areia e pedregulho das amostras.

\begin{tabular}{|c|c|c|c|c|}
\hline Amostra & Pedregulho (\%) & Areia (\%) & Silte (\%) & Argila (\%) \\
\hline$*$ AR (100\%) & - & 93,5 & 4,4 & 2,1 \\
\hline Solo (100\%) & 3,2 & 42,8 & 3,3 & 50,7 \\
\hline AR (25\%) + Solo & - & 56,0 & 4,0 & 40,0 \\
\hline AR (50\%) + Solo & - & 74,2 & 6,8 & 19,0 \\
\hline AR (75\%) + Solo & 0,1 & 88,5 & 5,6 & 5,8 \\
\hline
\end{tabular}

$* \mathrm{AR}=$ agregado reciclado

Fonte: Autor

Os resultados obtidos de Coeficiente de Uniformidade $(\mathrm{Cu})$ e da dimensão característica máxima do agregado reciclado classificaram este como bem graduado de acordo com a norma NBR 15115 (ABNT, 2004).

Para complementar a análise granulométrica, as amostras foram classificadas segundo o Sistema Unificado de Classificação de Solos (ASTM, 1990), como mostra o Quadro 3.

\section{Quadro 3 - Classificação dos materiais segundo o SUCS.}

\begin{tabular}{|c|c|}
\hline Materiais & Classificação SUCS \\
\hline Agregado reciclado (AR) & Areia bem graduada -SW \\
\hline Solo & Argila arenosa $-\mathrm{CL}$ \\
\hline $\operatorname{AR}(25 \%)+$ Solo & Areia argilosa - SC \\
\hline AR $(50 \%)+$ Solo & Areia siltosa $-\mathrm{SM}$ \\
\hline $\operatorname{AR}(75 \%)+$ Solo & Areia siltosa $-\mathrm{SM}$ \\
\hline
\end{tabular}

Os valores de densidade real das amostras ficaram compreendidos entre $2,63 \mathrm{~g} / \mathrm{cm}^{3} \mathrm{e}$ $2,69 \mathrm{~g} / \mathrm{cm}^{3}$.

Através dos ensaios de Limite de Liquidez (LL) e Limite de Plasticidade (LP) verificouse que tanto a mistura de AR (75\%) + Solo quanto o AR (100\%) não apresentaram LL e LP, comportando-se como materiais não plásticos.

\subsubsection{Forma dos grãos}

A Tabela 2 mostra os diferentes percentuais encontrados na amostra estudada para cada classificação de forma dos grãos, verificando que existe uma predominância de grãos cúbicos $(58 \%)$. Esta forma foi constatada também em outras pesquisas realizadas por Lima (2008) e Jiménez (2011), mostrando que essa forma provém, em parte, do processo de beneficiamento, no qual o agregado reciclado passou.

Tabela 2 - Forma dos grãos e as respectivas porcentagens do agregado reciclado.

\begin{tabular}{|c|c|}
\hline Classificação da forma & Presença no agregado reciclado (\%) \\
\hline Cúbica & 58,0 \\
\hline Alongada & 26,1 \\
\hline Lamelar & 15,2 \\
\hline Alongada-lamelar & 0,7 \\
\hline
\end{tabular}

Fonte: Autor

LIMA (2008) observou que os agregados com a forma lamelar tende a se quebrar mais facilmente quando sujeito a ações construtivas ou até mesmo ao tráfego, diferentemente 
dos materiais que apresentam a forma cúbica, que são mais indicados para pavimentação.

Os percentuais das diferentes formas encontradas no agregado em estudo atendem à NBR 15115 (ABNT, 2004), que recomenda para o agregado reciclado uma porcentagem de grãos lamelares inferior a $30 \%$ e com um percentual menor que $1 \%$ para as partículas com a forma alongada-lamelar.

\subsection{Caracterização mecânica}

\subsubsection{Compactação}

O ensaio de compactação foi realizado com reuso de materiais, caso contrário, iria precisar de mais material para a execução do mesmo. Este ensaio foi importante para a obtenção das curvas de compactação e dos parâmetros necessários para a realização dos ensaios de ISC.

Nas curvas de compactação das amostras de solo e de agregado recilado observou-se o formato de sino, o que é bem relevante, pois em função da grande variabilidade das amostras, os resultados com agregados reciclados são em geral bem dispersos, dificultando a obtenção da curva de compactação, LEITE, (2007); MOLENAAR e NIEKEERK, (2002).

A Tabela 3 apresenta os valores das umidades ótimas e dos pesos específicos dessas curvas. Observou-se que a umidade ótima das misturas variou entre 15,3\% e 18,6\%, mostrando uma redução à medida que o percentual de agregado reciclado aumentou. Em contrapartida, ocorreu o inverso com o peso específico seco máximo, aumentando com a maior variação de fração granular.

Tabela 3 - Umidades ótimas e pesos específicos dos materiais sem fibras.

\begin{tabular}{|c|c|c|}
\hline Materiais & $\mathbf{W}_{\text {ot }}(\mathbf{\%})$ & $\boldsymbol{\gamma}_{\mathbf{s}}\left(\mathbf{k N} / \mathbf{m}^{\mathbf{3}}\right)$ \\
\hline *AR (100\%) & 15,5 & 17,1 \\
\hline Solo (100\%) & 22,0 & 16,4 \\
\hline *AR (25\%)+ Solo & 18,6 & 18,0 \\
\hline *AR (50\%) + Solo & 16,4 & 18,7 \\
\hline *AR (75\%) + Solo & 15,3 & 19,4 \\
\hline
\end{tabular}

*AR = agregado reciclado

Fonte: Autor

Souza, Segantini e Pereira (2008) também observaram essa mesma tendência em relação aos parâmetros de compactação com a adição de resíduos no solo argiloso. Verificaram que o aumento do peso específico da mistura com a presença dos resíduos é devido a uma melhor acomodação das partículas durante a compactação. Com isto, o material torna-se mais atraente em vistas aos melhoramentos, como, por exemplo, ser mais compacto, mais resistente, com menor absorção de água e, consequentemente, mais durável.

\subsection{2 Índice de Suporte Califórnia}

Através dos ensaios de Índice de Suporte Califórnia, foram obtidos os resultados de ISC (com e sem imersão) e da expansão das amostras, como mostra a Tabela 4. Tais valores mostram que a presença do agregado reciclado nas misturas fez aumentar o ISC. Esse 
acréscimo resultou em um incremento de ISC superior a cinco vezes (com imersão) e seis vezes (sem imersão) em relação ao valor de ISC do solo.

Observou-se que os valores de ISC com imersão foram inferiores quando comparados com os valores obtidos sem imersão. Isso foi esperado, já que após $96 \mathrm{~h}$ de imersão a resistência do material saturado diminui devido à perda de sucção.

De acordo com os parâmetros relativos a NBR 15115 (ABNT, 2004), foi possível verificar que as amostras com e sem imersão de AR $(75 \%)+$ Solo podem servir para todas as camadas de um pavimento. A mistura de AR $(50 \%)+$ Solo serve tanto para sub-base quanto para reforço de subleito. Contudo, o solo e amostra com o menor percentual de agregado reciclado (25\%) na mistura podem ser utilizados apenas como reforço de subleito, já que apresentaram valores inferiores a $20 \%$ e superiores a $12 \%$, como indicado na norma.

Tabela 4 - Resultados de ISC, com e sem imersão, e da expansão dos materiais.

\begin{tabular}{|c|c|c|c|}
\hline \multirow{2}{*}{ Materiais } & \multicolumn{2}{|c|}{ Resultados desta pesquisa } & \multirow{2}{*}{ Expansão (\%) } \\
\cline { 2 - 3 } & Com imersão & Sem imersão & 0,00 \\
\hline *AR (100\%) & 79,1 & 93,9 & 0,32 \\
\hline Solo (100\%) & 12,9 & 14,1 & 0,06 \\
\hline AR (25\%) + Solo & 13,2 & 17,6 & 0,10 \\
\hline AR (50\%) + Solo & 32,9 & 42,2 & 0,03 \\
\hline AR (75\%) + Solo & 73,4 & 86,5 & \\
\hline
\end{tabular}

*AR = agregado reciclado

Fonte: Autor

\subsubsection{Avaliação da quebra dos grãos pós-ISC}

Para efeito de comparação, foi realizada a avaliação da quebra dos grãos após o ensaio de ISC e foi possível verificar pequenas variações na diferença de percentual de material passante nas curvas das misturas, com exceção da amostra de solo, na qual as variações das curvas com e sem imersão em relação a curva inicial (antes do ensaio de ISC) foram maiores.

A Tabela 5 mostra que os valores dos Índices de Degradação de Ruiz e o IDp do DNER - ME 398/99, com e sem imersão, foram muito próximos, não havendo também diferença significativa entre esses valores e os da quebra dos grãos após o ensaio de ISC.

Tabela 5 - Índices de Degradação de Ruiz (ID) e o IDp após o ISC.

\begin{tabular}{|c|c|c|c|c|}
\hline \multirow{2}{*}{ Amostra } & \multicolumn{2}{|c|}{ ID Ruiz após o ISC } & \multicolumn{2}{c|}{ IDp após o ISC } \\
\cline { 2 - 5 } & Sem imersão & Com imersão & Sem imersão & Com imersão \\
\hline *AR (100\%) & 4,90 & 5,38 & 4,54 & 4,66 \\
\hline Solo (100\%) & 11,47 & 11,35 & 10,15 & 11,03 \\
\hline AR (25\%)+ Solo & 3,48 & 3,84 & 3,48 & 3,93 \\
\hline AR (50\%) + Solo & 7,03 & 7,43 & 6,26 & 6,67 \\
\hline AR (75\%) + Solo & 4,92 & 5,01 & 4,07 & 4,55 \\
\hline
\end{tabular}

*AR = agregado reciclado

Fonte: Autor

As alterações nas curvas granulométricas são devido à quebra dos grãos durante o ensaio. Porém as amostras continuam atendendo a norma NBR 15115 (ABNT, 2004) 
em relação à porcentagem passante na peneira $0,42 \mathrm{~mm}$ e ao coeficiente de uniformidade.

\section{CONSIDERAÇÕES FINAIS}

Com base nos resultados obtidos na presente pesquisa, é possível mostrar as seguintes considerações:

As curvas granulométricas mostraram a distribuição das partículas de cada amostra, sendo o agregado reciclado classificado como um material bem graduado.

A forma dos grãos do agregado reciclado atende a norma NBR 15115 (ABNT, 2004), pois predominou a forma cúbica com $58 \%$, que são mais indicados para pavimentação. Além disso, os grãos lamelares foram inferiores a 30\% e a forma alongada-lamelar foi menor que $1 \%$.

As curvas de compactação apresentaram formato de sino, sendo muito inusitado devido à dificuldade de sua obtenção pela grande variabilidade do agregado reciclado.

As umidades ótimas das amostras apresentaram redução com o acréscimo de agregado reciclado, enquanto o peso específico seco máximo aumentou com a maior variação de fração granular.

Os valores de CBR mostram as aplicações em diferentes camadas de um pavimento que as amostras podem ser utilizadas.

A avaliação da quebra dos grãos pelos índices de Ruiz (ID) e do DNER-ME 398/99 (IDp) permitiu verificar através das curvas granulométricas que não houve variações significativas, apenas na amostra de solo.

Os valores desta pesquisa se enquadram no intervalo de valores obtidos por diversos autores, sendo considerados satisfatórios em camadas para pavimentos. Apesar da recomendação da NBR 15115 (ABNT, 2004) e da NBR 15116 (ABNT, 2004) de não utilizar agregado reciclado em vias de alto volume de tráfego, o material desta pesquisa poderia ser utilizado, já que o valor do ISC supera os $60 \%$ indicado pela norma.

\section{REFERÊNCIAS}

ASSOCIAÇÃO BRASILEIRA DE NORMAS TÉCNICAS (ABNT). NBR 6459: Solo Determinação do limite de liquidez. Rio de Janeiro, 1984.

. NBR 6508: Grãos de solos que passam na peneira de 4,8 mm - Determinação da massa especifica. Rio de Janeiro, 1984.

. NBR 6954: Lastro Padrão: determinação da forma do material. Rio de Janeiro, 1989.

. NBR 7180: Solo - Determinação do limite de plasticidade. Rio de Janeiro, 1984.

. NBR 7181: Solo - Análise Granulométrica - Procedimento. Rio de Janeiro, 1984.

. NBR 7182: Solo - Ensaio de Compactação - Procedimento. Rio de Janeiro, 1986.

. NBR 9895: Solo - Índice de suporte Califórnia. 14p. Rio de Janeiro, 1987.

. NBR 15.115: Agregados reciclados de resíduos sólidos da construção civil Execução de camadas de pavimentação - Procedimentos. Rio de Janeiro, 2004, 10 p.

. NBR 15116: Agregados reciclados de resíduos sólidos da construção civil Utilização em pavimentação e preparo de concreto sem função estrutural - Requisitos. Rio de Janeiro, 2004. 
AMERICAN SOCIETY FOR TESTING AND MATERIALS, (1990). Annual book of ASTM standards. Section 4: Construction, v.04.08: Soil and rock; dimension stone; geosynthetics. Philadelphia.

BUZZATTI , D.J. (1987). Indice de abrasão los angeles e índice de degradação: análise comparativa paradiversas naturezas de agregados. Departamento de estradas e rodagem do estado de Minas Gerais, Publicação técnica, № 23.

CORNELI, Vanessa Medeiros. Análise da gestão de resíduos de construção e demolição no município de Campo Mourão/Paraná Maringá 2009. 2009. 157 f. Dissertação (Mestrado) - Curso de Pós-graduação Em Engenharia Urbana, Departamento de Engenharia Civil, Universidade Estadual De Maringá, Maringá, 2009.

DEPARTAMENTO NACIONAL DE ESTRADAS DE RODAGEM. DNER - ME 398/99 Agregados: determinação do índice de degradação após compactação. Próctor IDP. Rio de Janeiro; 1999.

GUSMÃO, Alexandre Duarte. Manual de Gestão dos Resíduos da Construção Civil. Camaragibe, PE: CCS Gráfica, 2008. 140 p.

JIMÉNEZ, A. M. G. Estudo experimental de um resíduo de construção e demolição (RCD) para utilização em pavimentação. 2011. 123 f. Dissertação (Mestrado) - Curso de Mestrado em Geotecnia, Departamento de Engenharia Civil e Ambiental, Universidade de Brasília, Distrito Federal, 2011.

LAFAYETTE, K. P. V. Estudo Geológico-Geotécnico do Processo Erosivo em Encostas no Parque Metropolitano Armando de Holanda Cavalcanti - Cabo de Santo Agostinho/PE. 2006. 358 f. Tese (Doutorado em Engenharia Civil) - Programa de Pós-graduação em Engenharia Civil, Universidade Federal de Pernambuco, Recife. 2006.

LEITE, F. da C. Comportamento mecânico de agregado reciclado de resíduo sólido da construção civil em camadas de base e sub-base de pavimentos. 2007. 185 f. Dissertação (Mestrado) - Departamento de Engenharia de Transportes, Escola Politécnica da Universidade de São Paulo, São Paulo - SP, 2007.

LIMA, J. H. C. Utilização de Resíduo de Construção e Demolição para pavimentos urbanos da Região Metropolitana de Fortaleza. 2008. Dissertação de Mestrado, Programa de Mestrado em Engenharia de Transportes, Universidade do Ceará, Fortaleza, CE, $162 \mathrm{fl}$.

LIRA JR., E. A. de. Uso de resíduos de construção e demolição (RCD) em estacas de compactação para melhoramento de solos. Recife. UPE, 2010. 166f. Dissertação (Mestrado). Escola Politécnica da Universidade de Pernambuco. Programa de Pós-Graduação em Engenharia Civil. Recife.

MOLENAAR, A. A. A.; VAN NIEKERK, A. A. Effects of gradation, composition, and degree of compaction on the mechanical characteristics of recycled unbound materials.

Transportation Research Record, Washington, n.1787, p.73-82, 2002.

SILVA, E. P. da; COUTINHO, R. Q.; LIMA FILHO, M.; NEUMANN, V. H.; LIMA, R. P. de,. Avaliação do Processo Evolutivo e da Dinâmica Erosiva: Um Estudo de Caso no Parque Metropolitano Armando de Holanda Cavalcanti - Cabo de Santo Agostinho - PE /BRASIL. Estudos Geológicos. Série B, Estudos e Pesquisas (UFPE), v. 23, p. 53-75, 2013.

SOUZA, M.I.B.; SEGANTINI, A.S.S.; PEREIRA, J.A. 2008. Tijolos prensados de solocimento confeccionados com resíduos de concreto. Revista Brasileira de Engenharia Agrícola e Ambiental, v. 12, № 2, p. 205-212, 2008.

VALLE, N. Utilização de solos residuais saprolíticos na pavimentação rodoviária em Santa Catarina. Dissertação (Mestrado). Programa de Pós-Graduação em Engenharia Civil. Universidade Federal de Santa Catarina, Florianópolis, 1994. 